\title{
Cooperative Spontaneous Emission from Nanocrystals to a Surface Plasmon Polariton in a Metallic Nanowire
}

\author{
Joong-Hag Lee, Suc-Kyoung Hong, and Seog Woo Nam* \\ Department of Display and Semiconductor Physics, Korea University, Jochiwon 339-700, Korea \\ Seog-Seong Kim \\ Department of Physics, Chungbuk National University, Cheongju 361-763, Korea
}

(Received August 4, 2011 : revised September 14, 2011 : accepted September 19, 2011)

\begin{abstract}
We analyze the cooperative spontaneous emission of optically excited nanocrystals into surface plasmon polaritons propagating on the surface of a cylindrical metallic nanowire. The spontaneous emission probability of the nanocrystals is obtained by perturbative expansions with and without dipole-dipole interaction among nanocrystals in order to see the cooperative effects. The spontaneous emission probability depends on the radial and axial distributions, as well as on the dipolar orientation of nanocrystals. It is shown that the spontaneous emission probability is strongly influenced by dipole-dipole interaction, axial distribution, and dipolar orientation of nanocrystals for closely spaced nanocrystals.
\end{abstract}

Keywords: Plasmonic nanowire, Nanocrystals, Cooperative spontaneous emission

OCIS codes : (250.5403) Plasmonics; (260.2510) Fluorescence

\section{INTRODUCTION}

The optical properties of composite nanostructures including metal are strongly affected by surface plasmon polaritons' (SPPs) collective oscillations of conduction electrons with respect to the positive ion background [1-5]. SPPs are the propagating excitations of charge-density waves, and their associated intense electromagnetic fields are generated in the vicinity of the surface of a conductor. They are bound to and propagate along the metal/dielectric interface. Recently, great attention has been paid to the study of the strong coupling between individual emitters and SPPs in metallic nanowires [6-9]. The emission properties of nanoscale optical emitters can be significantly modified by the proximity of a nanowire that supports surface plasmons because an SPP is generally very intense and highly localized $[5,7,10]$. This subwavelength localization is accompanied by a dramatic condensation of optical fields. A substantial increase in the coupling strength $\mathrm{g} \propto 1 / \sqrt{V_{\text {eff }}}$ can be achieved near the surface of metal nanostructures, since the effective mode volume $V_{\text {eff }}$ of the SPPs can be considerably reduced. For an optical emitter placed within the evanescent SPP mode tail, the spontaneous emission probability of the excited energy of an emitter into the surface plasmons is tremendously enhanced [11-14]. Thus, the controllable strong near-field coupling between emitters and SPPs in metal nanostructures has stimulated a number of studies on nano-plasmonics [15-18].

Near-field coupling of excited colloidal nanocrystals (NCs) to SPPs in a metallic nanowire (NW) is a challenging problem since nanocrystals are randomly dispersed relative to the metallic nanowire [17]. In this paper, we investigate the spontaneous emission (SE) of the excited energy of one NC in NC aggregation to the SPPs in a nearby metallic nanowire for some specified configurations and for specific transition dipolar orientation of NCs. The radius of the NW is to be so small that it is sufficient to consider only the fundamental TE SPP mode in our calculations. In this work, we analyze the cooperative effect in the spontaneous decay of an excited $\mathrm{NC}$ in an NC aggregate into a SPP mode with and without the dipole-dipole interaction between NCs [8]. The spontaneous emission rate into surface plasmons can be suppressed or enhanced for specially arranged NCs, depending on their relative positions and transition dipolar

\footnotetext{
*Corresponding author: phynam@korea.ac.kr

Color versions of one or more of the figures in this paper are available online.
} 
orientations due to their quantum interference between the interactions. This variable emission rate can be characterized by cooperativeness, which for thin wires and closely spaced NCs is predicted to be large. In the next section, a theoretical modeling is employed to calculate the transition amplitude of the spontaneous emission under the perturbative expansion scheme. Results of the numerical evaluation for the transition probability (TP) of the spontaneous emission are presented in Sec. III followed by a discussion on its characteristics. A brief conclusion is given in Sec. IV.

\section{THEORETICAL MODEL}

The system of interest in this paper is schematically shown in Fig. 1. NCs are supposed to be aggregated around the silver nanowire along the axis, where only one of the NCs is excited. First, we briefly review the properties of SPPs propagating along the surface of metallic NW [19, 20], which have been observed experimentally [21-24]. We are especially interested in azimuthally independent SPPs, which are supported under the condition that the radius of the wire is small enough such that only the fundamental TM surface plasmon polariton mode exists while all other higher order modes are cut off [7]. In this case the electric and magnetic fields of the fundamental TM surface plasmon polariton mode are given by [19]

$$
\begin{aligned}
\vec{E}_{q}\left(k_{z} ; \rho, z, t\right) & =a_{q}\left(k_{z}\right)\left(-\frac{i k_{z}}{K_{q}} \psi_{1, q}\left(K_{q} \rho\right) \hat{\rho}+\psi_{0, q}\left(K_{q} \rho\right) \hat{z}\right) \\
& \times e^{i k_{z} z} e^{-i \omega t}, \\
\vec{H}_{q}\left(k_{z} ; \rho, z, t\right) & =a_{q}\left(k_{z}\right) \frac{-i \varepsilon_{q} \omega}{c^{2} \mu_{q} K_{q}} \psi_{1, q}\left(K_{q} \rho\right) \hat{\varphi} e^{i k_{z} z} e^{-i \omega t},
\end{aligned}
$$

where $q$ stands for inside or outside of the metallic NW with

$$
\begin{aligned}
& K_{q}^{2}=\frac{\omega^{2} \varepsilon_{q}(\omega)}{c^{2}}-k_{z}^{2}, \\
& \psi_{n, \text { in }}\left(K_{i} \rho\right)=J_{n}\left(K_{\text {in }} \rho\right), \\
& \psi_{n, \text { out }}\left(K_{o} \rho\right)=H_{n}^{(1)}\left(K_{\text {out }} \rho\right) .
\end{aligned}
$$

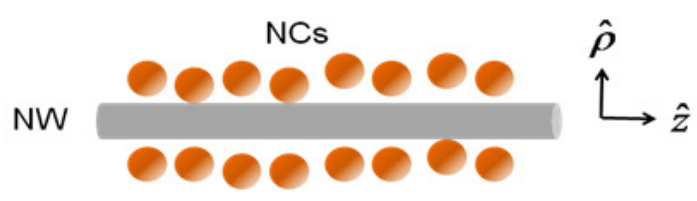

FIG. 1. Schematic view of the system. NCs are located in the vicinity of silver nanowire. Cylindrical coordinates are used with $z$ axis as the wire axis.
Here $J_{n}$ and $H_{n}^{(1)}$ are Bessel and Hankel function, respectively. The dielectric function $\varepsilon_{q}(\omega)$ is taken from the DrudeLorentz model, i.e., $\epsilon_{i n}(\omega)=\epsilon_{\infty}\left[1-\omega_{p}^{2} / \omega(\omega+i \gamma)\right]$, where $\gamma$ is the relaxation rate due to ohmic loss. Since the system does not have azimuthal dependence, the wave number of the SPP has only $z$ dependence. From now on we drop the subscript $\mathrm{z}$ for the wave number $k_{z}$. The dispersion relation $\left(k, \omega_{k}\right)$ of the SPP mode is obtained by applying the boundary condition of the TM mode fields on the surface of the metallic NW $[8,11,19,20]$

$$
\frac{(\omega / c)^{2} \varepsilon_{i}}{\mu_{i} K_{i} a} \frac{J_{0}{ }^{\prime}\left(K_{i} a\right)}{J_{0}\left(K_{i} a\right)}-\frac{(\omega / c)^{2} \varepsilon_{o}}{\mu_{o} K_{o} a} \frac{H_{0}^{(1)}\left(K_{o} a\right)}{H_{0}^{(1)}\left(K_{o} a\right)}=0,
$$

where $a$ is the radius of the NW. The dispersion relation for the fundamental mode of SPP on metallic NW is shown in Fig. 2. The mode-expansion coefficients $a_{q}(k)$ in Eqs. (1) and (2) are determined by matching the boundary condition of the fields on the metal nanowire/dielectric interface and also by normalizing the electromagnetic fields to their vacuum fluctuation energies $[1,8,11]$. After performing these operations, the resulting electric field outside the metal becomes

$$
\begin{aligned}
\vec{E}(k ; \rho, z, t) & =\sqrt{\frac{\hbar \omega}{\varepsilon_{0} 2 \pi L}} b(k)\left[-\frac{i k}{K_{q}} \psi_{1}\left(K_{q} \rho\right) \hat{\rho}+\psi_{0}\left(K_{q} \rho\right) \hat{z}\right] \\
& \times e^{i k z} e^{-i \omega t}
\end{aligned}
$$

where $b(k)$ is again the mode expansion coefficient but normalized by electric permittivity of the vacuum and nanowire length $L$, which is averaged over the azimuthal angle for the wire. The radial component of the field is (almost) real and the axial component of the field is imaginary, so that they are out of phase by $\pi / 2$. The absolute value of the

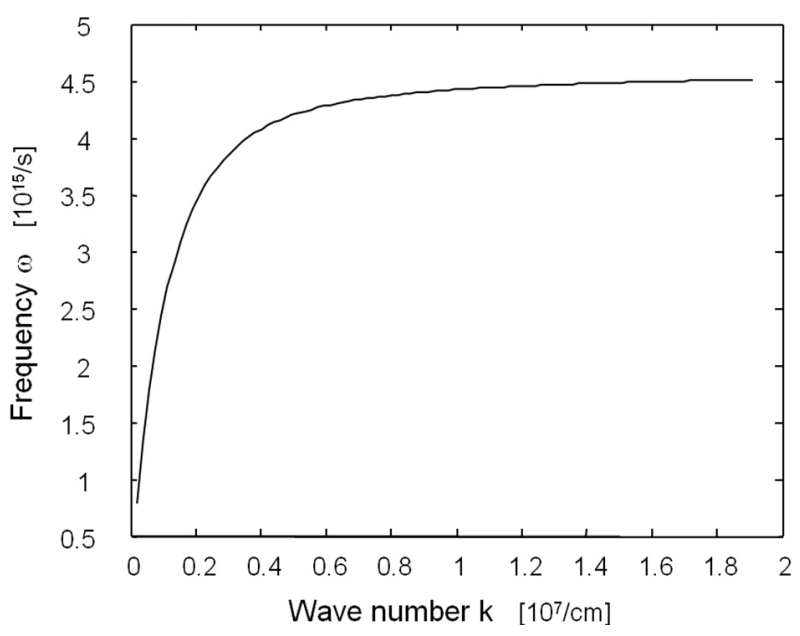

FIG. 2. Dispersion relation of the fundamental SPP mode on lossless silver, where $\epsilon_{\infty}=9.6$ for silver, $\epsilon_{\infty}=5.3$ for dielectric $(\mathrm{GaN}), \omega_{p}=3.76 \mathrm{eV} / \mathrm{h}, \gamma=0$, and the radius of the NW is set to be $a=5 \mathrm{~nm}$ 


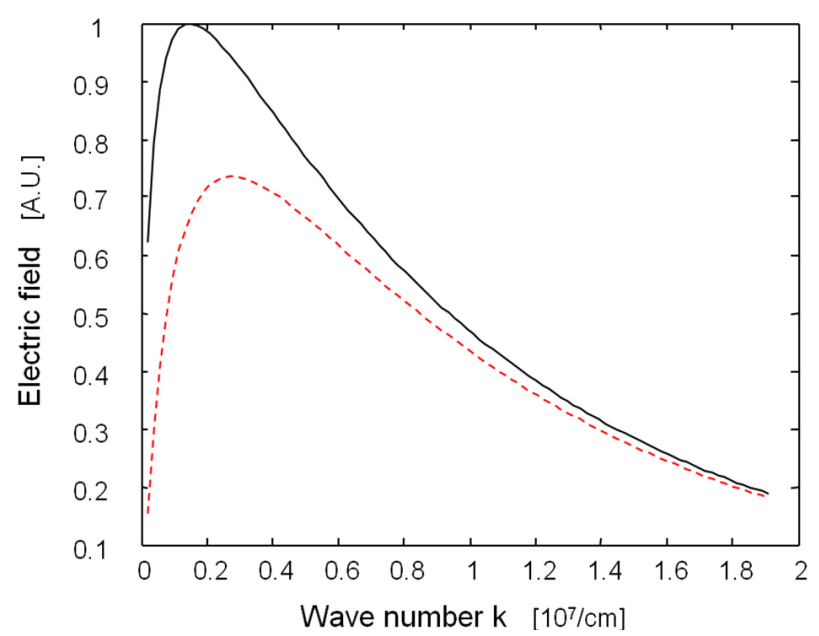

(a)

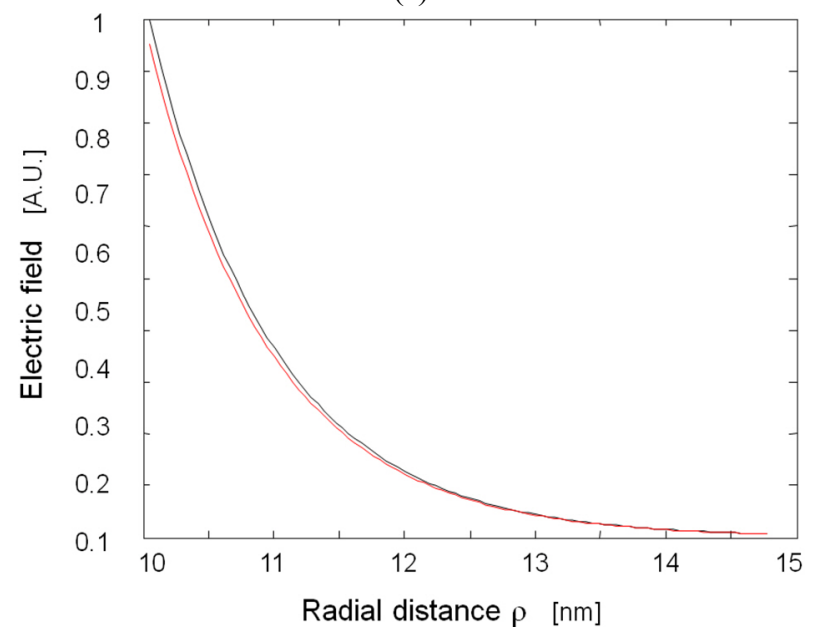

(b)

FIG. 3. Electric field outside the wire. (a) as a function of wave number for $\rho=1.2 a$ and (b) as a function of radial distance from the wire center for $k=10^{7} / \mathrm{cm}$. Arbitrary unit is used for vertical axis. The solid and dashed lines are for the radial and axial components of the field, respectively. The radial component is real and the axial component is imaginary.

electric field outside the nanowire as a function of wave number is shown in Fig. 3(a); solid and dashed lines are for the radial and axial components of the field, respectively. Fig. 3(b) is the electric field as a function of radial distance from the wire center. Dramatic concentration of optical fields is well manifested.

The Hamiltonian of this composite system is written by $H=H_{0}+H_{\text {int, }}$, where $H_{0}$ is the unperturbed part and the interaction $H_{\text {int }}$ is given by

$$
H_{\mathrm{int}}=\sum_{m}\left(-\vec{\mu}_{m} \cdot \vec{E}_{m}\right)+\sum_{m \neq j} \frac{\vec{\mu}_{m} \cdot \vec{\mu}_{j}-3\left(\vec{\mu}_{m} \cdot \hat{r}_{m j}\right)\left(\vec{\mu}_{j} \cdot \hat{r}_{m j}\right)}{r_{m j}^{3}},
$$

where $\vec{\mu}_{j}$ is the transition dipole moment of the $j$ th $\mathrm{NC}$ and $\vec{E}_{j}$ is the electric field at the location of the $j$ th $\mathrm{NC}$ due to the surface plasmon polaritons in the nanowire. $r_{m j}$ and $\hat{r}_{m j}$ are the distance and the directional unit vector between $m$ th and $j$ th $\mathrm{NCs}$, respectively. The first term represents the interaction between NCs and NW plasmons, while the second term describes the dipole-dipole interaction (DDI) between NCs. The transition energy of an NC is assumed to be detuned from the surface plasmon polarion mode energy of the metallic nanowire.

The transition amplitude of the SE is calculated from perturbation theory. Within the dipole approximation, the first order transition amplitude of the spontaneous emission from an excited $j$ th $\mathrm{NC}$ at $\left(\rho_{j}, z_{j}\right)$ with transition energy $\omega_{j}$ to a fundamental SPP of mode with wave number $k$ in metallic NW is obtained as

$$
c_{j \rightarrow k}^{(1)}(t)=\frac{1}{\hbar} g_{j, k}{ }^{*} e^{-i k z_{j}} \frac{e^{i \Delta_{k j} t}-1}{\Delta_{k j}},
$$

where $g_{j, k}=\vec{\mu}_{j} \cdot \vec{E}_{j, k}$ and $\Delta_{k j}=\omega_{k}-\omega_{j}$ with $\vec{E}_{j, k}$ being the electric field of the surface plasmon mode of wave number $k$ at the location of the jth NC. $\Delta_{k j}$ describes the detuning between $j$ th NC's transition energy and SPP mode energy with wave number $k$. If all NCs are identical with $\omega_{m}=\omega_{j}$ and the direct DDI between NCs is ignorable, the third order perturbative transition amplitude of the SE is calculated as

$$
\begin{aligned}
& c_{j \rightarrow k}^{(3)}(t)=\frac{i}{\hbar^{3}} \sum_{m} g_{m, k}{ }^{*} e^{-i k z_{m}} \sum_{k^{\prime}} g_{m, k^{\prime}} g_{j, k^{\prime}}{ }^{*} e^{-i k^{\prime}\left(z_{j}-z_{m}\right)} \\
& \times\left[\frac{-t e^{i \Delta_{k j} t}}{\Delta_{k^{\prime} j} \Delta_{k j}}+\left(\frac{1}{i \Delta_{k^{\prime} j}^{2}}+\frac{1}{i \Delta_{k^{\prime} j} \Delta_{k j}}\right) \frac{e^{i \Delta_{k j} t}-1}{\Delta_{k j}}-\frac{1}{\Delta_{k^{\prime} j}{ }^{2}} \frac{e^{i \Delta_{k k^{\prime}} t}-1}{i \Delta_{k k^{\prime}}}\right]
\end{aligned}
$$

The even-order perturbation terms are null, since the coupling is linear to the dipole moment. Since the NC's transition energy is detuned from the SPPs in the metallic nanowire and $\sin \left(\Delta_{k k^{\prime}} t / 2\right) / \Delta_{k k^{\prime}}$ is a very strongly peaked function of $\Delta_{k k^{\prime}}$, the last term dominates. Thus Eq. (10) can be approximated by

$$
\begin{aligned}
c_{j \rightarrow k}^{(3)}(t) \approx & -\frac{1}{\hbar^{3}} \sum_{m} g_{m, \vec{k}} \sum_{k^{\prime}} g_{m, \vec{k}} g_{j, \vec{k}^{\prime}}{ }^{*} \\
& \times e^{-i k z_{m}-i k^{\prime}\left(z_{j}-z_{m}\right)} \frac{1}{\Delta_{k^{\prime} j}{ }^{2}} \frac{e^{i \Delta_{k k^{\prime},}}-1}{\Delta_{k k^{\prime}}} .
\end{aligned}
$$

When all NCs are not identical, the third order perturbative transition amplitude of the SE is similarly calculated, but in this case the detuning parameter $\Delta_{m j}$ between NCs is, of course, explicitly included in the expression 


$$
\begin{aligned}
& c_{j \rightarrow k}^{(3)}(t)=\frac{-1}{\hbar^{3}} \sum_{m} g_{m, k}{ }^{*} e^{-i k z_{m}} \sum_{k^{\prime}} g_{m, k^{\prime}} g_{j, k^{\prime}}{ }^{*} e^{-i k^{\prime}\left(z_{j}-z_{m}\right)} \\
& \times\left[\frac{1}{\Delta_{k^{\prime} j} \Delta_{m j}} \frac{e^{i \Delta_{k^{\prime} j^{\prime}} t}-1}{\Delta_{k j}}+\left(\frac{1}{\Delta_{k^{\prime} j} \Delta_{k^{\prime} m}}-\frac{1}{\Delta_{k^{\prime} j} \Delta_{m j}}\right) \frac{e^{i \Delta_{k m^{\prime}} t}-1}{\Delta_{k m}}-\frac{1}{\Delta_{k^{\prime} j} \Delta_{k^{\prime} m}} \frac{e^{-i \Delta_{k^{\prime} k} t}-1}{\Delta_{k^{\prime} k}}\right]
\end{aligned}
$$

On the other hand, if the direct DDI between NCs cannot be ignored, the second order transition amplitude of the SE term survives. From the calculation carried out for the case when all NCs are identical with only the nearest neighbor interaction, the transition amplitude of the SE is

$$
\begin{aligned}
c_{j \rightarrow k}^{(2)}(t)= & \frac{1}{\hbar^{2}}\left[g_{j+1, k} e^{-i k z_{j+1}} J_{(j+1) j}+g_{j-1, k} e^{-i k z_{j-1}} J_{(j-1) j}\right] \\
& \times\left[\frac{e^{i \Delta_{k j} t} t}{i \Delta_{k j}}+\frac{e^{i \Delta_{k j} t}-1}{\Delta_{k j}{ }^{2}}\right]
\end{aligned}
$$

where $J_{m j}$ is the dipole-dipole interaction energy between $m$ th and $j$ th NCs in Eq. (8). Similarly, when all NCs are not identical, the second order transition amplitude of the $\mathrm{SE}$ is calculated as

$$
\begin{aligned}
c_{j \rightarrow k}^{(2)}(t)= & \frac{-1}{\hbar^{2}} g_{j+1, k}{ }^{*} e^{-i k z_{j+1}} J_{(j+1) j} \frac{1}{\Delta_{(j+1) j}}\left(\frac{e^{i \Delta_{k j} t}-1}{\Delta_{k j}}-\frac{e^{i \Delta_{k(j+1)} t}-1}{\Delta_{k(j+1)}}\right) \\
& +\frac{1}{\hbar^{2}} g_{j-1, k}{ }^{*} e^{-i k z_{j-1}} J_{(j-1) j} \frac{1}{\Delta_{j(j-1)}}\left(\frac{e^{i \Delta_{k j} t}-1}{\Delta_{k j}}-\frac{e^{i \Delta_{k(j-1)}}-1}{\Delta_{k(j-1)}}\right)
\end{aligned}
$$

\section{NUMERICAL EVALUATION AND DISCUSSION}

The first order TP, $P_{k}^{(1)}(t)$ for the SE of an excited NC to a SPP of mode $k$ does not exhibit any collective effect, since it only involves single NC coupling to SPP mode regardless of the existence of other NCs. In order to see the collective effect, the higher order perturbative terms must be examined. We first consider the case without DDI. When we evaluate the total TP composed of $c_{j \rightarrow k}^{(1)}(t)$ and $c_{j \rightarrow k}^{(3)}(t)$, the TP would have the cross product contributions between the first and third order transition amplitudes of the SEs. However, neither do they show any substantial collective effect on the same footing as the first order contribution alone. Then, the most important contribution with respect to the cooperative effect comes from the square of $c_{j \rightarrow k}^{(3)}(t)$. From Eq. (10), the third order TP for the SE of an NC to an SPP of mode $k, P_{k}^{(3)}(t)$ is obtained by

$$
\begin{aligned}
& P_{k}^{(3)}(t)=\frac{1}{\hbar^{6}} \sum_{n, m, k^{\prime}, k^{\prime \prime}}\left|g_{n, k} g_{n, k^{\prime \prime}} g_{m, k} g_{m, k^{\prime}} g_{j, k^{\prime}} g_{j, k^{\prime \prime}}\right| \\
& \exp \left(i \Phi_{n, m, k^{\prime}, k^{\prime \prime}}\right) f\left(k, k^{\prime}, k^{\prime \prime}, t\right),
\end{aligned}
$$

where $g_{j, k}=\left|g_{j, k}\right| e^{i \theta_{j, k}}$ and

$$
\begin{aligned}
& \Phi_{n, m, k^{\prime}, k^{n}}=k\left(z_{n}-z_{m}\right)+k^{\prime}\left(z_{m}-z_{j}\right)+k^{\prime \prime}\left(z_{j}-z_{n}\right)+ \\
& \theta_{n, k}-\theta_{m, k}+\theta_{m, k^{\prime}}-\theta_{j, k^{\prime}}+\theta_{j, k^{n}}-\theta_{n, k^{n}} .
\end{aligned}
$$

Here, $f\left(k, k^{\prime}, k^{\prime \prime}, t\right)$ is the absolute square of the temporal part in Eq. (10) and it is a function of $\Delta_{k 0}, \Delta_{k^{\prime} 0}, \Delta_{k^{\prime \prime} 0}, \Delta_{k k^{\prime}}$, and $\Delta_{k k}$, so that it represents the consequence of detuning effect. As seen from Eq. (15), characteristic collective behavior of this composed system in the third order TP is actually described by the phase factor $\exp \left(i \Phi_{n, m, k^{\prime}, k^{\prime \prime}}\right)$.

The cooperativeness in the third order TP is manifested by the phase $\Phi_{n, m, k^{\prime}, k^{\prime}}$, which describes the correlation among the whole NC aggregate. Since the mode of SPPs does not depend on the azimuthal angle, the phase $\Phi_{n, m, k^{\prime}, k^{\prime \prime}}$ of Eq. (16) depends only on the radial and axial locations of NCs. Thus, the cooperativeness is determined by the radial and axial arrangement of NCs, i.e., the locations of $\operatorname{NCs}\left(\rho_{j}, z_{j}\right)$ and by the dipolar orientation. In order to analyze the collective effect, we consider the dependence of the phase factor on the radial and axial arrangement as well as on the dipolar orientation of NCs. First, the phase difference caused by the axial distribution of NCs in the form of $k\left(z_{n}-z_{m}\right)$ in Eq. (16) is always non-negligible since $k \Delta z \sim \pi$ even for $\Delta z \sim O(1 \mathrm{~nm})$. Thus the axial distribution of NCs along the wire can be an important factor to induce the collective effect in the SE. Next, for the analysis of the phase caused by the radial arrangement and the dipolar orientation, which is represented by the term of $\theta_{j, k}$ in Eq. (16), we consider, for simplicity, the case when the transition dipole vector is in the plane of $(\rho, z)$,. In this case, the absolute magnitude of the NC-SPP coupling strength $\left|g_{j, k}\right|$ and its phase $\theta_{j, k}$ are expressed as

$$
\left|g_{j, k}\right|=\mu_{j}\left|\cos \alpha_{j}\right| \sqrt{\left|E_{\rho, k}\left(\rho_{j}\right)\right|^{2} \tan ^{2} \alpha_{j}+\left|E_{z, k}\left(\rho_{j}\right)\right|^{2}}
$$

and

$$
\theta_{j, k}=\tan ^{-1}\left(\frac{\left|E_{z, k}\left(\rho_{j}\right)\right|}{\left|E_{\rho, k}\left(\rho_{j}\right)\right| \tan \alpha_{j}}\right),
$$

respectively, where $\alpha_{j}$ is the orientation of the transition dipole measured from the wire axis. $\left|g_{j, k}\right|$ and $\theta_{j, k}$ as functions of $k$ and $\alpha_{j}$ are shown in Fig. 4(a) and 4(b), respectively. 
Now we consider the collective effect of the SE in the third order TP. As was pointed out in the previous section, the terms with $\left(\sin \Delta_{k k^{\prime}} t / 2\right) / \Delta_{k k^{\prime}}$ and $\left(\sin \Delta_{k k^{\prime \prime}} t / 2\right) / \Delta_{k k^{\prime \prime}}$ in $f\left(k, k^{\prime}, k^{\prime \prime}, t\right)$ of Eq. (15) dominate, since NC's transition energy is detuned from the SPPs in the metallic NW. The amplitude of the function $\left(\sin \Delta_{k k^{\prime}} t / 2\right) / \Delta_{k k^{\prime}}$, which is very highly peaked at $\Delta_{k k^{\prime}}=0$, increases as $t$ and decreases to zero when $\Delta_{k k^{\prime}}=2 \pi / t$. When time $t \sim z_{n}(d k / d \omega)$ is small enough as $\sim f s$ or less, non-resonant coupling between SPP modes is allowed in the third order process. In this situation, the phase $\Phi$ of Eq. (16) is not negligible. That is, the phase difference with the form of $z_{n}\left(k-k^{\prime}\right)$ in Eq. (16) is order of $\pi$ for the time being $\sim f_{s}$ or less in which $\Delta z$ is assumed to be $\pm 10 \mathrm{~nm}$. On the other hand, in the longtime limit, $\Delta_{k k^{\prime}} \approx \Delta_{k k^{\prime \prime}} \approx 0$ and for the most part only the resonant coupling between SPP modes is allowed in the third order process. In this circumstance, there are many cancellations among the phases in Eq. (16), so that no appreciable collective effect is expected. In this resonant coupling approximation, the third order transition probability of the SE can be approximated as

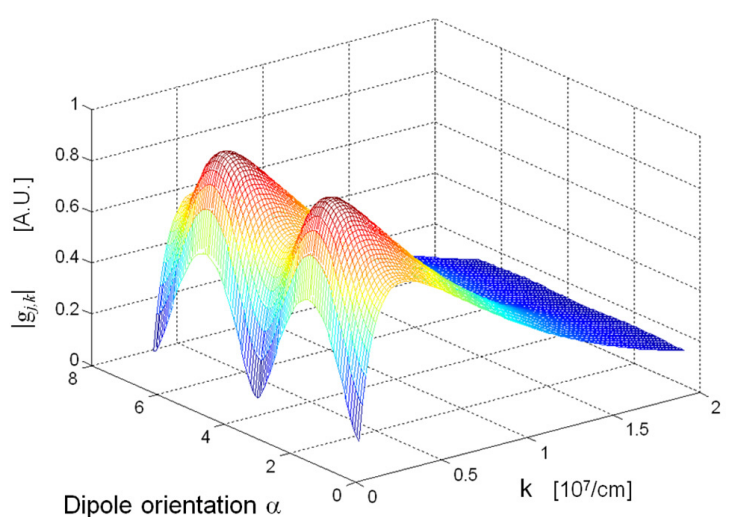

(a)

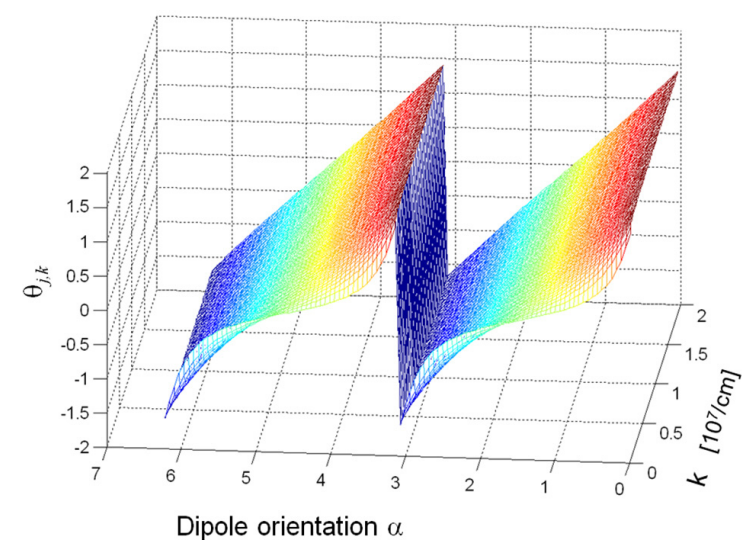

(b)

FIG. 4. NC-SPP coupling constant $g_{j, k}$. (a) The coupling strength $\left|g_{j, k}\right|$ as a function of the dipolar orientation and the wave number for $\rho=1.2 a$. (b) The phase of the coupling constant $\theta_{j, k}$ as a function of the dipolar orientation $\alpha$ and the wave number $k$.

$$
P_{k}^{(3)}(t) \approx \frac{L}{\hbar^{6}}\left(\frac{d k}{d \omega}\right) \frac{t}{\Delta_{k j}{ }^{4}}\left|g_{j, k}\right|^{2} \sum_{m, n}\left|g_{m, k}\right|^{2}\left|g_{n, k}\right|^{2},
$$

which is plotted as a function of $k$ in Fig. 5(a). Since the transition energy of an NC is assumed to be detuned from the SPP mode energy of the NW and to be above the mode energy, the TP of the SE will increase as the size of NCs increases. That is, when the size of NCs increases, the transition energy of NCs decreases and then $\Delta_{k j}$ in Eq. (19) will decrease. In the case of non-identical NCs, it can be further approximated as $P_{k}^{(3)}(t)\left(1+\Delta_{j m} / \Delta_{k j}\right)^{-2}$, which shows reduced transition probability of the SE for increased disparity in the size of NCs. As a reference, the first order TP of the SE to the $k$ mode of SPP $P_{k}^{(1)}(t)$ is given by

$$
P_{k}^{(1)}(t)=\frac{4}{\hbar^{2}}\left|g_{j, k}\right|^{2} \frac{\sin ^{2} \Delta_{k j} t / 2}{\Delta_{k j}^{2}},
$$

and is shown in Fig. 5(b), for comparison. No noticeable

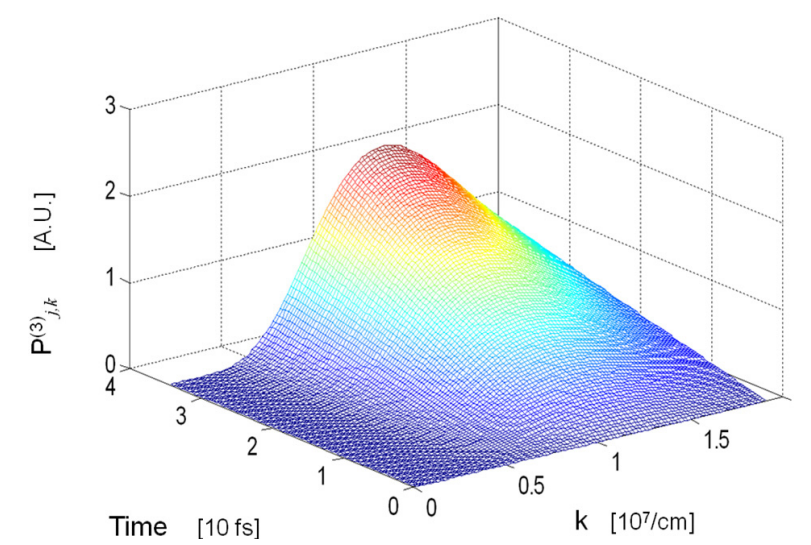

(a)

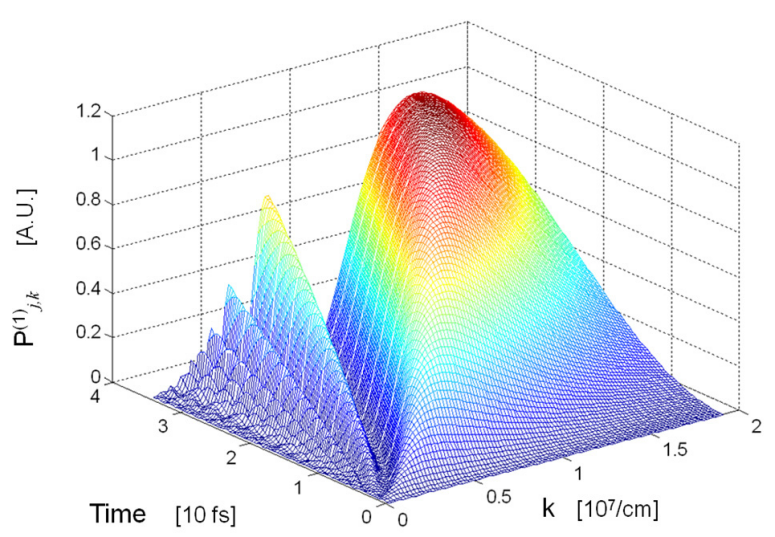

(b)

FIG. 5. Transition probability of the spontaneous emission to the k mode of SPP without temporal part as a function of time and wave number for $\rho=1.2 a$. (a) The third order transition probability of the SE under resonant approximation. (b) The first order transition probability of the SE. 
change for TP is observed with regard to including multiple interactions between NC and SPP.

Now we analyze the cooperative effect due to direct DDI. When all NCs are identical, the second order TP of the SE to the $k$ mode of SPP, $P_{k}^{(2)}(t)$ is obtained as

$$
\begin{aligned}
P_{k}^{(2)}(t) & =\frac{1}{\hbar^{4} \Delta_{k j}{ }^{2}}\left(\left|g_{j+1, k} J_{(j+1) j}\right|^{2}+\left|g_{j-1, k} J_{(j-1) j}\right|^{2}\right. \\
& \left.+2\left|g_{j+1, k} g_{j-1, k}\right| J_{(j+1) j} J_{(j-1) j} \cos \Phi\right) \\
& \times\left(t^{2}-2 t \frac{\sin \Delta_{k j} t}{\Delta_{k j}}+\frac{4 \sin ^{2} \Delta_{k j} t / 2}{\Delta_{k j}{ }^{2}}\right),
\end{aligned}
$$

where

$$
\Phi_{j, k}=k\left(z_{j+1}-z_{j-1}\right)+\theta_{(j-1) k}-\theta_{(j+1) k} .
$$

The cooperativeness of NCs is involved in the phase $\Phi$ as the case without DDI. As described above, the phase difference caused by the axial distance between NCs, is order of $\pi$ for $\Delta \mathrm{z} \sim 10 \mathrm{~nm}$. On the other hand, the phase difference, $\Delta \theta=\theta_{m, k}-\theta_{n, k}$ is induced by the radial distribution and the dipolar orientation of NCs, as seen from Eq. (18). Since the radial component of the electric field is (almost) real and the axial component of the electric field is imaginary, there is no interference effect coming from the NC-SSP coupling difference as seen from Eq. (18), if the transition dipole is arranged only along the wire or only perpendicular to the wire axis. Thus the phase difference $\Delta \theta$ in Eq. (22) depends strongly on the dipolar orientation. This character is plotted as a function of $\alpha$ and $k$ in Fig. 6(a). The figure shows that, for a given transition dipolar orientation of NCs, the SE of an excited NC into several specified modes of the fundamental SPP in the metallic nanowire is totally inhibited.

When the radial distances of NCs from the wire center are almost the same among the $\mathrm{NC}$ aggregates $\left(\left|g_{m, k}\right| \approx\left|g_{k}\right|\right)$ and the orientations of NC's transition dipoles are parallel to each other with DDI coupling $J$, the transition probability of the SE is approximated as

$$
P_{k}^{(2)} \approx \frac{4 J^{2}\left|g_{k}\right|^{2}}{\hbar^{4} \Delta_{k j}{ }^{2}} \cos ^{2} \frac{\Phi}{2} f(t) .
$$

In this case, the phase difference $\Delta \theta$ of Eq. (22) almost vanishes and the phase is determined by the difference of axial distance between NCs, which is plotted as a function of $\Delta z$ and $k$ in Fig. 6(c). The effect of interference on the SE due to the second order TP caused by the direct DDI is explicitly shown in the figure. For a given configuration of NCs, the SE to a certain specified SPP modes is totally

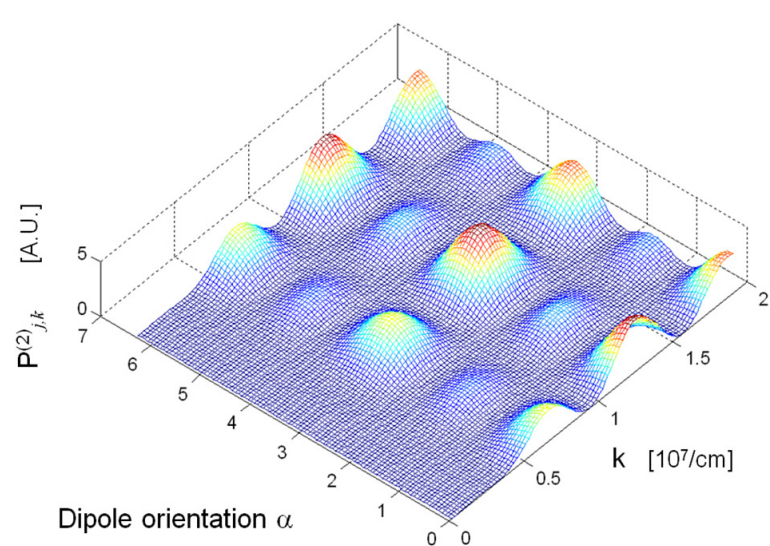

(a)

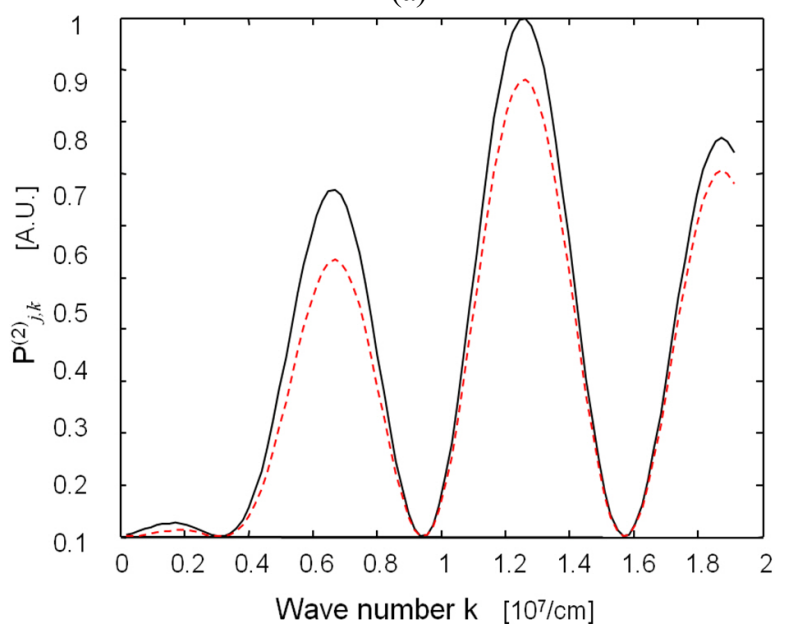

(b)

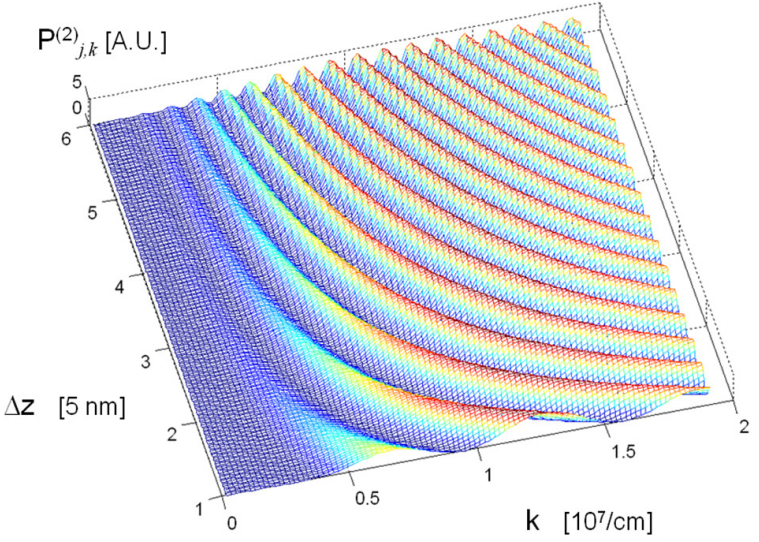

(c)

FIG. 6. The second order transition probability of the SE to the $\mathrm{k}$ mode of SPP when all DDI coupling is the same and dipolar directions are all the same, where $\Delta z=z_{j+1}-z_{j}=a$, and $f(t)$ is set to be unity. (a) TP as a function of $k$ and $a$. (b) TP as a function of for $a=0$. Solid and dashed lines are for $\hat{\mu}_{m}$ $=\hat{\rho}$ and $\hat{\mu}_{m}=\hat{z}$, respectively. (c) TP as a function of $k$ and $\Delta z$ for $a=\pi / 4$. The range of the inter-NC distance $\Delta z$ is from a to $6 a$.

suppressed, as expected. And when all NCs are arranged 
along the NW, the TP of the SE will decrease as the distance between the NCs and the NW increases as seen from Fig. 3(b).

In practice, the phase difference $\Delta \theta$ caused by the radial distribution between NCs is appreciable, however the effect of the radial distribution of $\mathrm{NC}$ on the cooperativeness in the second order TP is negligible due to the huge difference of the field $\left|E\left(\rho_{n}\right)\right|-\left|E\left(\rho_{m}\right)\right|$, which causes low visibility for the interference pattern of Eq. (21). Since the phase difference $\Delta \theta$ depends strongly on the dipolar orientation, the effect of dipolar orientation on the cooperativeness in the second order TP is considered by dealing with two simple cases. For instance, when there are three NCs with similar radial distances $\rho_{j} \approx \rho$ and if $\hat{r}_{m j}=\hat{z}, \hat{\mu}_{1}=\hat{\rho}, \hat{\mu}_{2} \cdot \hat{z}$ $=\cos \theta$, and $\hat{\mu}_{3}=\hat{z}$, the second order TP as a function of $\alpha$ and $k$ is obtained as shown in Fig. 7(a). On the other hand, if the first and the third dipoles point along the radial direction, i.e., $\hat{\mu}_{1}=\hat{\mu}_{3}=\hat{\rho}$, the transition probability of the $\mathrm{SE}$ as a function of $\alpha$ and $k$ behaves like Fig. 7(b). Here, we have analyzed the TP of the SE when only one $\mathrm{NC}$ is

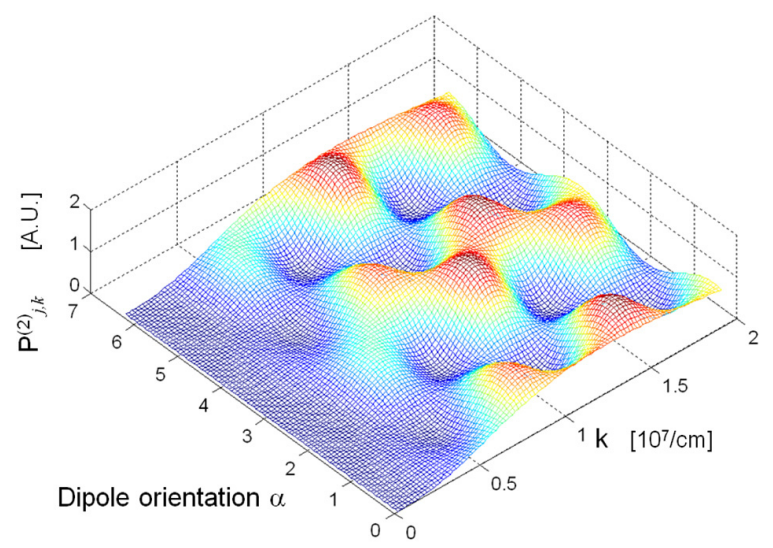

(a)

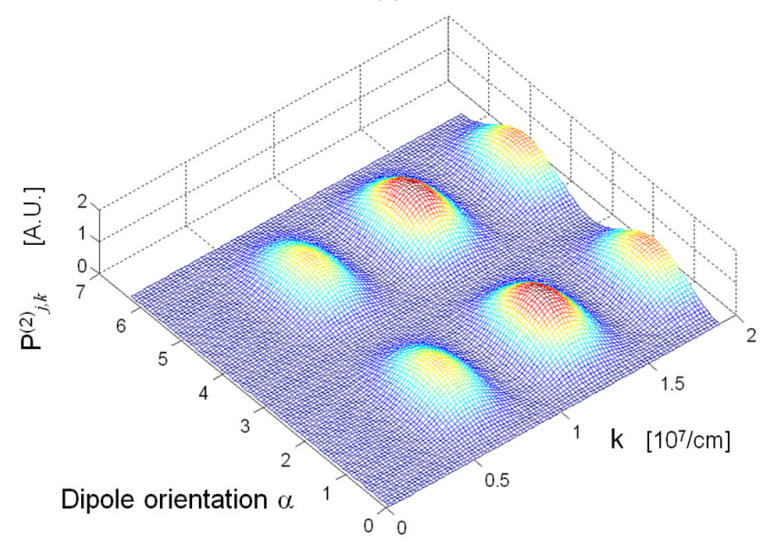

(b)

FIG. 7. The second order transition probability of the SE to the k mode of SPP when $\rho_{j} \approx \rho$ with $\hat{r}_{m j}=\hat{z}, \hat{\mu}_{1}=\hat{\rho}, \hat{\mu}_{2} \cdot \hat{z}=$ $\cos \theta$ and $\Delta z=a$. (a) TP as a function of $k$ and $\alpha$ when $\hat{\mu}_{3}=\hat{z}$. (b) TP as a function of $k$ and $\alpha$ when $\hat{\mu}_{3}=\hat{\rho}$. excited. Meanwhile, if two or more NCs are excited, then the TP of the SE will strongly depend on the initially prepared state. For the case without the interaction between excitons, if the NCs are excited in the form of a superradiative (subradiative) state, the emission rate will be enhanced (suppressed) [25].

\section{CONCLUSION}

In summary, we have analyzed the characteristics of the cooperative feature of the spontaneous emission of one excited NC among NC aggregate into a mode of SPP in a metallic nanowire. In order to see the collective effect of the SE, higher order perturbation expansions of the transition probability of the SE have been derived. In particular, the influence of the dipolar orientation and the axial and radial arrangement of NCs has been considered. The first-order transition probability of the SE does not show collective behavior. Also the collective effect disappears in the thirdorder process of the spontaneous emission when direct DDI is not involved under the resonant coupling approximation between SPPs. However, the second-order transition probability of the SE yields a cooperative effect of the SE. In particular, the spontaneous emission of the excited $\mathrm{NC}$ energy is suppressed or enhanced depending on the relative orientation of the dipoles and the arrangement of NCs.

\section{ACKNOWLEDGMENT}

This work has been supported by a Korea University Grant.

\section{REFERENCES}

1. S. A. Maier, Plasmonics: Fundamentals and Applications (Springer, New York, USA, 2007), Chapter 1.

2. H. Wang, D. W. Brandl, P. Nordlander, and N. J. Halas, "Plasmonic nanostructures: artificial molecules," Acc. Chem. Res. 40, 53-62 (2007).

3. A. I. Fernandez-Dominguez, L. Martin-Moreno, F. J. GarciaVidal, S. R. Andrews, and S. A. Maier, "Spoof surface plasmon polariton modes propagating along periodically corrugated wires," IEEE J. Quantum Electron. 14, 1515-1521 (2008).

4. J. Yang, Q. Cao, and C. Zhou, "Analytical recurrence formula for the zeroth-order metal wire plasmon of terahertz waves," J. Opt. Soc. Am. A 27, 1608-1612 (2010).

5. H. Wei, D. Ratchford, X. Li, H. Xu, and C.-K. Shih, "Propagating surface plasmon induced photon emission from quantum dots," Nano Lett. 9, 4168-4171 (2009).

6. H. Ditlbacher, A. Hohenau, D. Wagner, U. Kreibig, M. Rogers, F. Hofer, F. R. Aussenegg, and J. R. Krenn, "Silver nanowires as surface plasmon resonators," Phys. Rev. Lett. 95, 257403 (2005).

7. D. E. Chang, A. S. Sørensen, P. R. Hemmer, and M. D. Lukin, "Strong coupling of single emitters to surface plasmons," 
Phys. Rev. B 76, 035420 (2007).

8. M.-T. Cheng, Y.-Q. Luo, Y.-Y. Song, and G.-X. Zhao, "Plasmonic waveguides mediated energy transfer between two distant quantum dots," J. Mod. Opt. 57, 2177-2181 (2010).

9. F. L. Kien, S. D. Gupta, K. P. Nayak, and K. Hakuta, "Nanofiber-mediated radiative transfer between two distant atoms," Phys. Rev. B 72, 063815 (2005).

10 D. E. Chang, A. S. Sørensen, E. A. Demler, and M. D. Lukin, "A single-photon transistor using nanoscale surface plasmons," Nat. Phys. 3, 807-812 (2007).

11. Y. N. Chen, G. Y. Chen, D. S. Chuu, and T. Brandes, "Quantum-dot exciton dynamics with a surface plasmon: band-edge quantum optics," Phys. Rev. A 79, 033815 (2009).

12. A. V. Akimov, A. Mukherjee, C. L. Yu, D. E. Chang, A. S. Zibrov, P. R. Hemmer, H. Park, and M. D. Lukin, "Generation of single optical plasmons in metallic nanowires coupled to quantum dots," Nature 450, $402-406$ (2007).

13. G.-Y. Chen, Y.-N. Chen, and D.-S. Chuu, "Spontaneous emission of quantum dot excitons into surface plasmons in a nanowire," Opt. Lett. 33, 2212-2214 (2008).

14. I. D. Rukhlenko, D. Handapangoda, M. Premaratne, A. V. Fedorov, A. V. Baranov, and C. Jagadish, "Spontaneous emission of guided polaritons by quantum dot coupled to metallic nanowire: beyond the dipole approximation," Opt. Express 17, 17570-17581 (2009).

15. D. Witthaut and A. S. Sorensen, "Photon scattering by a three-level emitter in a one-dimensional waveguide," New J. Phys. 12, 043052 (2010).

16. D. Y. Lei, A. Aubry, S. A. Maier, and J. B. Pendry,
"Broadband nano-focusing of light using kissing nanowires," New J. Phys. 12, 093030 (2010).

17. D. M. O'Carroll, C. E. Hofmann, and H. A. Atwater, "Conjugated polymer/metal nanowire heterostructure plasmonic antennas," Adv. Mater. 22, 1223-1227 (2010).

18. S. John and T. Quang, "Spontaneous emission near the edge of a photonic band gap," Phys. Rev. A 50, 1764-1769 (1994).

19. J. A. Stratton, Electromagnetic Theory (McGraw-Hill, New York, USA, 1941), Chapter 9.

20. C. A. Pfeiffer, E. N. Economou, and K. L. Ngai, "Surface polaritons in a circularly cylindrical interface: surface plasmons," Phys. Rev. B 10, 3038-3051 (1974).

21. G. Schider, J. R. Krenn, A. Hohenau, H. Ditlbacher, A. Leitner, F. R. Aussenegg, W. L. Schaich, I. Puscasu, B. Monacelli, and G. Boreman, "Plasmon dispersion relation of Au and Ag nanowires," Phys. Rev. B 68, 155427 (2003).

22. E. J. R. Vesseur, R. de Waele, M. Kuttge, and A. Polman, "Direct observation of plasmonic modes in $\mathrm{Au}$ nanowires using high-resolution cathodoluminescence spectroscopy," Nano Lett. 9, 2843-2846 (2007).

23. M. A. Schmidt and P. St. J. Russell, "Long-range spiralling surface plasmon modes on metallic nanowires," Opt. Express 16, 13617-13623 (2008).

24. A. W. Sanders, D. A. Routenberg, B. J. Wiley, Y. Xia, E. R. Dufresne, and M. A. Reed, "Observation of plasmon propagation, redirection, and fan-out in silver nanowires," Nano Lett. 6, 1822-1826 (2006).

25. S.-K. Hong, S. W. Nam, and K.-H. Yeon, "Coherent exciton states of excitonic nanocrystal-molecules," Phys. Rev. B 76, 115330 (2007). 